\title{
CANDIDATES FOR EXTREME CARBON STARS
}

\author{
Kevin Volk, ${ }^{1}$ Sun KwoK, and Philip P. LANGILl \\ Department of Physics and Astronomy, University of Calgary, Calgary, Alberta, Canada T2N 1N4 \\ Received 1991 September 16; accepted 1991 November 21
}

\begin{abstract}
A group of candidates for extreme carbon stars has been identified based on their IRAS low-resolution spectra being similar to those of AFGL 3068 and IRC $+10^{\circ} 216$, which are prototypes of highly evolved carbon stars with very high mass-loss rates. Ground-based photometry has been obtained for five of these sources in order to determine the peaks of their energy distributions. All five sources have color temperatures between 300 and $500 \mathrm{~K}$, and have energy distributions very similar to AFGL 3068. The observed data are fitted with radiative transfer models with optical depths at $11.3 \mu \mathrm{m}$ ranging from 1.4 to 8 . These results suggest that these stars have among the most optically thick circumstellar envelopes of all carbon stars observed to date.
\end{abstract}

Subject headings: circumstellar matter - infrared: stars - stars: carbon

\section{INTRODUCTION}

As the result of the Two Microns (Neugebauer \& Leighton 1969) and Air Force (Price \& Walker 1976) infrared sky surveys, a number of carbon stars with unusually thick circumstellar dust shells have been discovered. The best known examples are IRC $+10^{\circ} 216$ and AFGL 3068, both of which have circumstellar envelopes that are rich in molecules (Olofsson 1987). The optical photospheres of these stars are very heavily obscured by the surrounding dust shells, to such an extent that it is difficult to obtain an optical spectrum. Such carbon stars (and OH/IR stars which are their oxygen-rich counterparts) radiate strongly in the far-infrared and their energy distributions typically peak around $20 \mu \mathrm{m}$. The large amount of circumstellar reddening causes these stars to be very faint in the visible, and they cannot be detected using optical objectiveprism surveys. Even at wavelengths around $1 \mu \mathrm{m}$ where a number of carbon stars have been discovered, stars similar to IRC $+10^{\circ} 216$ and AFGL 3068 would be too faint to detect. Thus, few examples of such stars are known.

The Infrared Astronomical Satellite (IRAS) provides the observations necessary to find more extreme carbon stars. The IR $A S$ broad-band observations at $12,25,60$, and $100 \mu \mathrm{m}$ cover the spectral region where such stars emit most of their energy. If IRC $+10^{\circ} 216$ is at a distance of $\sim 150 \mathrm{pc}$ (Leahy, Kwok, \& Arquilla 1987), then it is simple to show that IRAS could in principle detect similar sources out to a distance of at least 10 $\mathrm{kpc}$ even in the Galactic plane. However, because of confusion with other sources, particularly oxygen-rich AGB stars with circumstellar dust shells, it is impossible to identify candidates for extreme carbon stars based on the $12 \mu \mathrm{m}$ flux alone. Even when color ([12]-[25] and [25]-[60]) information is available, it is difficult to identify extreme carbon stars uniquely. Based on the colors of the prototypes IRC $+10^{\circ} 216$ and AFGL 3068, extreme carbon stars can be expected to fall near the blackbody line in a $12 / 25 / 60$ color-color diagram, with [12][25] $>1$ (see Walker et al. 1989 for a discussion of color-color diagram analysis). However, there are other types of sources (e.g., oxygen-rich stars, $\mathrm{H}$ in regions) that have overlapping colors in this region of the color-color diagram.

\footnotetext{
${ }^{1}$ National Fellow 1989-1991, Canadian Institute for Theoretical Astrophysics.
}

In order to distinguish extreme carbon stars from oxygenrich stars and $\mathrm{H}$ II regions, spectroscopic data are necessary. The most useful data available for such a search are the lowresolution 8-23 $\mu \mathrm{m}$ spectra obtained by the IRAS mission (hereafter the LRS spectra). Oxygen-rich stars and $\mathrm{H}$ II regions generally show silicate features in their LRS spectra and can be readily separated from carbon stars. The extreme carbon stars that we are seeking can also be distinguished from the more normal carbon stars with thinner circumstellar envelopes. While the LRS of normal carbon stars show either a featureless photospheric continuum or the $11.3 \mu \mathrm{m} \mathrm{SiC}$ dust emission feature, extreme carbon stars have very weak (e.g., IRC $+10^{\circ} 216$ ) or no (e.g., AFGL 3068) SiC feature, and have a relatively low temperature blackbody-like continuum. Several candidates of extreme carbon stars have been independently identified in this manner by Omont et al. (1990). In this paper we report a systematic search of candidates for extreme carbon stars based on the LRS data, as well as results from groundbased observations of five such candidates.

\section{A SAMPLE OF EXTREME CARBON STARS}

We have identified a sample of extreme carbon stars in the Atlas of Low Resolution IRAS Spectra (1986, hereafter the LRS Atlas) that possess the following spectral characteristics:

1. The continuum must be relatively flat compared with either ordinary stars or most carbon stars. Specifically, the ratio of the shortest wavelength flux density $\left(\lambda F_{\lambda}\right)$ to the longest wavelength flux density in the LRS spectrum has to be less than 4.

2. There must be either no recognizable dust emission feature or only a relatively weak SiC emission feature in the LRS spectrum.

3. The spectrum should show a downturn near $8 \mu \mathrm{m}$ as is seen in the LRS spectrum of AFGL 3068. Since such a downturn is only seen in carbon stars, it is safe to assume that this is a real spectral feature.

4. The source should not be associated with a bright star, and preferably should have no association with cataloged objects except the AFGL catalog (Price \& Walker 1976).

Sources with very low-temperature continua, such that the LRS spectrum rises from 8 to $23 \mu \mathrm{m}$ in a $\lambda$ versus $\lambda F_{\lambda}$ plot, were excluded from the sample. These sources, numbering 
TABLE 1

ir $A S$ Properties of the 31 Candidates for Extreme Carbon Stars

\begin{tabular}{|c|c|c|c|c|c|c|c|c|}
\hline \multirow[b]{2}{*}{ IRAS NAME } & \multicolumn{4}{|c|}{ IRAS Flux DENSITIES (Jy) } & \multirow[b]{2}{*}{ QUALITY } & \multicolumn{2}{|c|}{ Classification } & \multirow[b]{2}{*}{ Notes } \\
\hline & $12 \mu \mathrm{m}$ & $25 \mu \mathrm{m}$ & $60 \mu \mathrm{m}$ & $100 \mu \mathrm{m}$ & & Atlas & VC & \\
\hline $00210+6221 \ldots \ldots \ldots$ & 48.7 & 51.89 & 12.54 & 23.16 & 3331 & 12 & $\mathbf{U}$ & 1 \\
\hline $01144+6658 \ldots \ldots \ldots$ & 139.90 & 206.1 & 64.67 & 15.85 & 3333 & 21 & $\mathbf{U}$ & \\
\hline $02293+5748 \ldots \ldots \ldots$ & 173.00 & 159.5 & 42.18 & 9.86 & 3333 & 42 & $\mathrm{C}$ & \\
\hline $02345+5422 .$. & 33.85 & 35.85 & 10.90 & 2.44 & 3333 & 23 & $\mathrm{U}$ & 2 \\
\hline $03313+6058 \ldots$ & 30.87 & 43.44 & 15.08 & 6.40 & 3333 & 22 & $\mathrm{U}$ & \\
\hline $03448+4432 \ldots$ & 130.30 & 93.87 & 21.86 & 6.03 & 3333 & 42 & $\mathrm{C}$ & \\
\hline $04530+4427 \ldots \ldots \ldots$ & 100.20 & 77.99 & 19.27 & 6.13 & 3333 & 42 & $\mathrm{C}$ & \\
\hline $06012+0726 \ldots \ldots \ldots$ & 319.60 & 225.6 & 55.90 & 15.45 & 3333 & 22 & $\mathrm{C}$ & \\
\hline $06582+1507 \ldots \ldots \ldots$ & 38.67 & 55.53 & 17.29 & 4.50 & 3333 & 22 & $\mathrm{U}$ & \\
\hline $08074-3615 \ldots \ldots \ldots$ & 155.90 & 124.8 & 33.54 & 7.66 & 3333 & 22 & $\mathrm{U}$ & \\
\hline $08171-2134 \ldots \ldots \ldots$ & 119.50 & 129.2 & 38.87 & 10.72 & 3333 & 22 & $\mathrm{U}$ & \\
\hline $08544-4431 \ldots \ldots \ldots$ & 180.30 & 158.8 & 56.25 & 28.43 & 3321 & 21 & $\mathrm{C}$ & 3 \\
\hline $09178-5556 \ldots \ldots \ldots$ & 15.45 & 10.16 & 2.56 & 3.62 & 3331 & 14 & $\mathrm{U}$ & \\
\hline $12042-6355 \ldots \ldots \ldots$ & 47.29 & 63.35 & 18.47 & 75.73 & 3331 & 11 & $\mathrm{U}$ & \\
\hline $12195-6830 \ldots \ldots \ldots$ & 33.89 & 30.89 & 7.72 & 3.58 & 3331 & 42 & C & 3 \\
\hline $12419-6058 \ldots \ldots \ldots$ & 68.22 & 75.14 & 17.84 & 40.77 & 3331 & 21 & $\mathrm{U}$ & \\
\hline $12550-7407 \ldots \ldots \ldots$ & 13.75 & 12.22 & 3.56 & 1.51 & 3333 & 13 & $\mathrm{U}$ & \\
\hline $12595-6035 \ldots \ldots \ldots$ & 15.53 & 11.40 & 5.93 & 14.07 & 3332 & 14 & $\mathrm{C}$ & \\
\hline $15471-5644 \ldots \ldots \ldots$ & 146.10 & 207.8 & 55.25 & 15.92 & 3333 & 21 & $\mathrm{U}$ & \\
\hline $17371-3021 \ldots \ldots \ldots$ & 114.70 & 95.62 & 23.42 & 191.1 & 2321 & 42 & $\mathrm{C}$ & 3 \\
\hline $17375-3652 \ldots \ldots \ldots$ & 125.70 & 196.3 & 49.47 & 61.07 & 3331 & 14 & $\mathrm{C}$ & 1,3 \\
\hline $17534-3030 \ldots \ldots \ldots$ & 196.50 & 228.6 & 70.57 & 34.50 & 3333 & 21 & $\mathrm{U}$ & \\
\hline $18464-0656 \ldots \ldots \ldots$ & 83.98 & 78.92 & 18.75 & 77.62 & 3331 & 21 & $\mathbf{U}$ & \\
\hline $19075+0921 \ldots \ldots \ldots$ & 133.00 & 163.7 & 54.91 & 78.02 & 3331 & 12 & $\mathrm{U}$ & 1 \\
\hline $19238+1159 \ldots \ldots \ldots$ & 53.03 & 59.27 & 18.68 & 76.91 & 3331 & 12 & $\mathbf{U}$ & 1 \\
\hline $19304+2529 \ldots \ldots \ldots$ & 37.09 & 49.50 & 12.64 & 7.03 & 3331 & 12 & $\mathbf{U}$ & \\
\hline $19548+3035 \ldots \ldots \ldots$ & 75.06 & 109.0 & 46.68 & 14.16 & 3333 & 21 & $\mathbf{U}$ & \\
\hline $19594+4047 \ldots \ldots \ldots$ & 338.40 & 260.1 & 59.86 & 17.38 & 3333 & 42 & C & \\
\hline $21318+5631 \ldots \ldots \ldots$ & 256.80 & 312.0 & 90.00 & 25.42 & 3333 & 21 & $\mathbf{U}$ & \\
\hline $21489+5301 \ldots \ldots \ldots$ & 110.90 & 93.40 & 26.77 & 7.99 & 3333 & 42 & $\mathrm{C}$ & \\
\hline $22303+5950 \ldots \ldots \ldots$ & 54.46 & 68.41 & 20.39 & 24.60 & 3331 & 41 & $\mathbf{U}$ & 1 \\
\hline $23166+1655 \ldots \ldots \ldots$ & 706.70 & 775.6 & 248.5 & 73.70 & 3333 & 02 & U & (AFGL 3068) \\
\hline
\end{tabular}

NoTES. (1) The LRS spectrum may have interstellar dust absorption near $10 \mu \mathrm{m}$. (2) The classification of the LRS spectrum is uncertain. It might be noisy silicate emission. (3) The $11.3 \mu \mathrm{m}$ feature is weak or otherwise questionable.

about 10 in all, are extremely interesting in their own right but are certainly different than AFGL 3068.

The application of the above criteria yielded 31 candidates. Eleven of these have weak $\mathrm{SiC}$ features and have IRAS [12][25] color greater than 1.2. The remaining 20 sources have unusually flat, nearly featureless LRS spectra judged to resemble the LRS spectrum of AFGL 3068. The 31 sources in this sample, plus AFGL 3068, are listed in Table 1. The table lists the IRAS Point Source Catalog (Version 2, 1988, herafter PSC) name, the flux densities $\left(F_{v}\right)$ in Jy (without any color corrections), the quality flags for these four bands $(3=$ good, $2=$ fair, $1=$ upper limit), the classification of the LRS spectrum from the LRS Atlas, and a letter classification of the LRS using the scheme of Volk \& Cohen (1989). The sources are either classified as group $\mathrm{C}$ ( $\mathrm{SiC}$ emission) or group $\mathrm{U}$ (unusual). The sources with the U classification are the best candidates for carbon stars similar to AFGL 3068, while those with $\mathrm{SiC}$ emission are more likely to be intermediate cases more similar to IRC $+10^{\circ} 216$ than to AFGL 3068. In a few cases the classification of the LRS spectrum is doubtful. These are listed in the notes following Table 1.

\section{INFRARED SPECTRAL CHARACTERISTICS}

The LRS spectra of the 21 group U sources in Table 1 are plotted in Figure 1. The plots give the IRAS name, the name of any associated object according the IRAS PSC, the letter clas- sification (all $U$ ), and the number classification from the LRS Atlas. In the LRS Atlas classification scheme, they are generally classified as type 11-15 (featureless, flat continuum) or type 21-23 (weak silicate emission). Those classified as having silicate emission all appear to be bad classifications, with the possible exception of $02345+5422$ where the spectrum may be an unusual example of silicate emission although there is no sign of the $18 \mu \mathrm{m}$ emission feature. Several of the sources $(22303+5950,19238+1159,00210+6221$, and $12419-6058)$ have what seems to be a weak silicate absorption feature at 10 $\mu \mathrm{m}$. This may be due to interstellar reddening, indicating distances of a few kpc for the sources. However, the expected 18 $\mu \mathrm{m}$ absorption is not observed. A weak $11.3 \mu \mathrm{m}$ emission feature may be present in the LRS spectrum of $08074-3615$.

A number of these 31 extreme carbon star candidates have previously been studied by ground-based observations. Most of the observations are molecular line observations, and the 15 sources in Table 1 that have been detected in $\mathrm{CO}$ are listed in Table 2. Since most infrared carbon stars show circumstellar $\mathrm{CO}$ emission, the $\mathrm{CO}$ detections are consistent with them being evolved carbon stars. Six of the candidates have sufficient ground-based infrared data to determine their energy distributions, and these are plotted in Figures 2 and 3 (see $\S 4$ ). A summary of existing infrared data on these candidates is given below.

$00210+6221$. - This source was observed in the midinfrared by Kwok, Hrivnak, \& Boreiko (1987). It is identified 

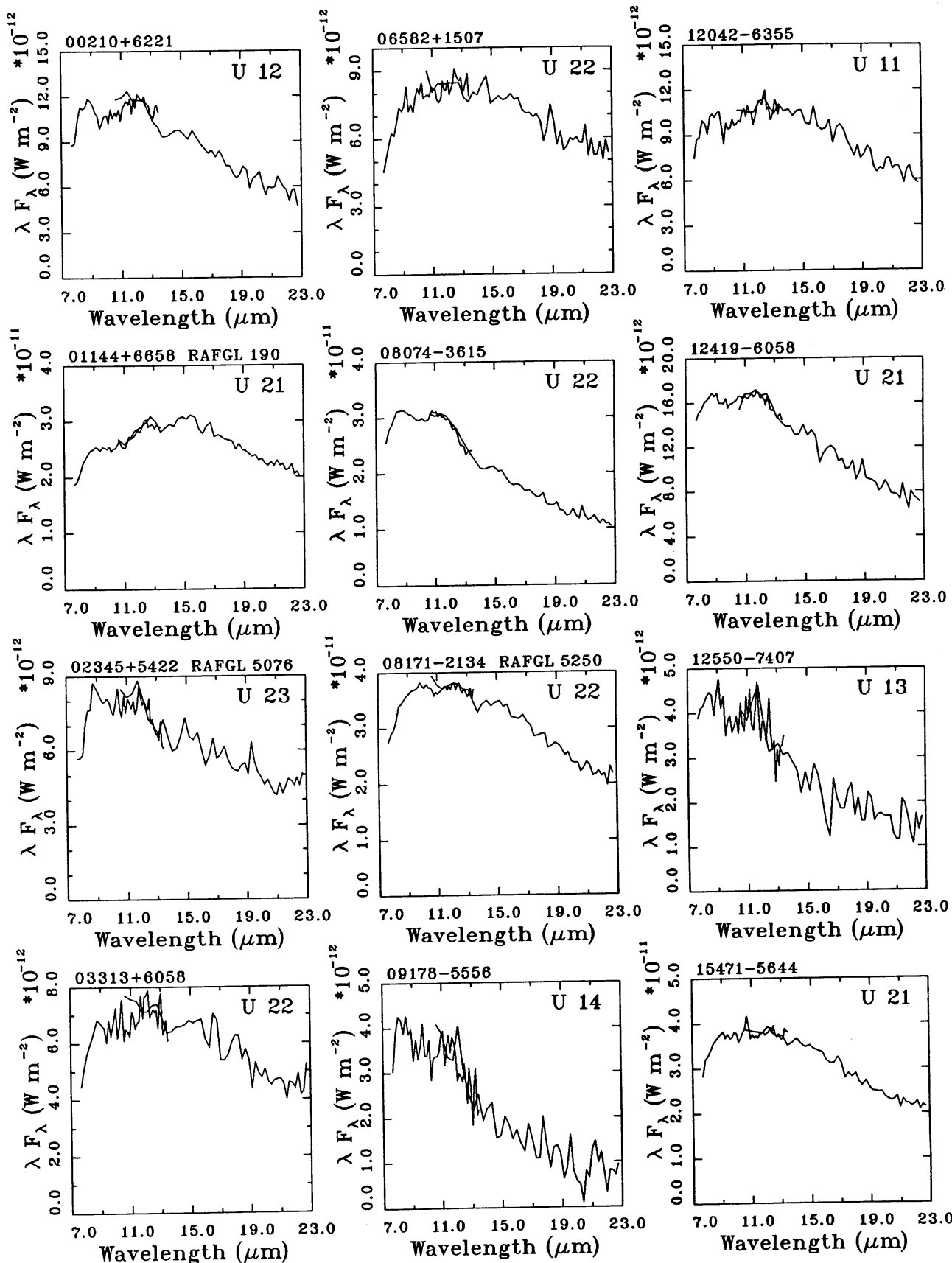

FIG. 1.-LRS spectra of 21 extreme carbon star candidates. On the top right corner are the two-digit LRS Atlas classification and the letter code (U) classification in the scheme of Volk \& Cohen (1989).

with a bright optical counterpart (Hrivnak, Kwok, \& Boreiko 1985). Optical photometry $(B=18.27, V=16.51, I=14.36)$ was obtained at the USNO by $H$. Harris (private communication). The bright optical counterpart of this object makes it unlikely to be an extreme carbon star. The energy distribution of this object is shown in Figure $2 a$.

$01144+6658$ ( $A F G L$ 190). - Near and mid-infrared observations have been made by Gerhz \& Hackwell (1976) and Lebofsky et al. (1978). The energy distribution is shown in Figure $2 b$.

$02293+5748(A F G L 341)$. - Classified as carbon-rich based on its infrared spectrum by Kleinmann, Gillett, \& Joyce (1981). Near and mid-infrared observations have been made by Gehrz \& Hackwell (1976). Their observations lie below both the IR $A S$ data and the observations at $N$ and $Q$ discussed in $\S 4$. This could be the result of source variability.

$06012+0726$ (AFGL 865). - Near-infrared observations of AFGL 865 have been carried out by Gaylard et al. (1989) and Jones et al. (1990). Near-infrared spectrophotometry obtained by Merrill \& Stein (1976) shows the $3.1 \mu \mathrm{m}$ absorption band commonly seen in optical carbon stars. Thus this source is certainly a carbon star. The available photometry shows that 

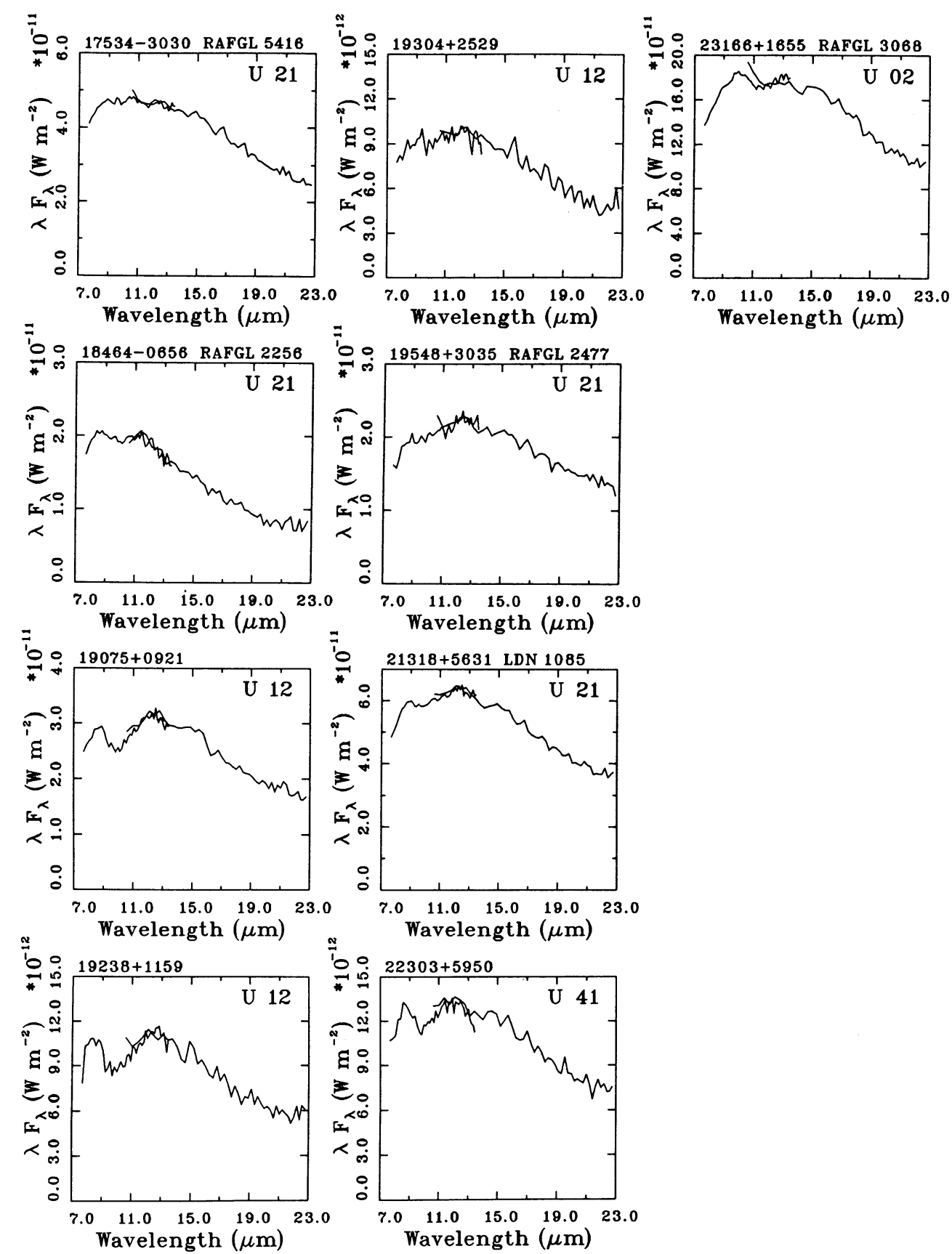

FIG. 1-Continued

AFGL 865 is variable with an amplitude of about 1.5 mag at $L$. The $L$-magnitude from Gaylard et al. (1989) is +3.43 compared with a range from 2.1 to 3.5 reported by Jones et al. (1990) and the value 1.94 obtained by us (see $\S 4$ ). The magnitude of the variability appears to be larger at $K$ than at $L$. Gaylard et al. (1989) give a $K$-magnitude of 7.74 compared with a range from 5.8 to 7.4 given by Jones et al. (1990). A period of 686 days for AFGL 865 was derived by Jones et al. (1990).

$19548+3035$ (AFGL 2477).-This source was found to have an optical counterpart by Gosnell, Hudson, \& Puetter (1979) and Hrivnak et al. (1985). This fact makes it unlikely to be an extreme carbon star. Figure $2 c$ shows the energy distribution of this object. There is a clear excess in the near infrared, which is typical of post-AGB objects in transition to planetary nebulae (Volk \& Kwok 1989). The relative weakness of the photospheric component suggests that it has just recently left the AGB.

$19594+4047$ (AFGL 2494). - This source was identified as a carbon star by Merrill \& Stein (1976). It was also observed in the infrared by Low et al. (1976), Joyce et al. (1977) and Jones et al. (1990). The energy distribution is shown in Figure $2 d$. A period of 783 days was derived from these observations. It is likely to be a carbon-rich Mira variable. 


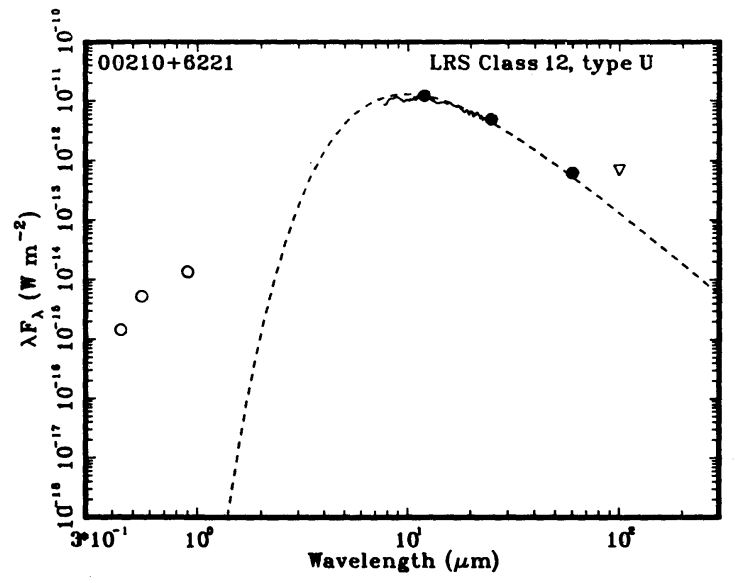

FIG. $2 a$

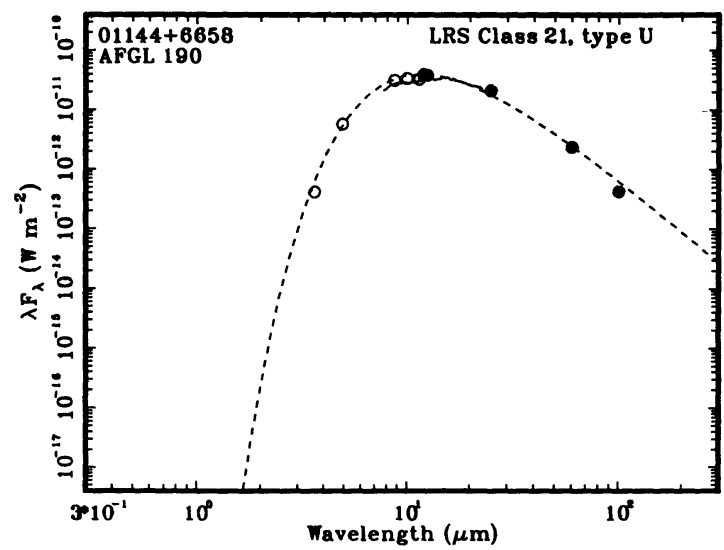

FIG. $2 c$

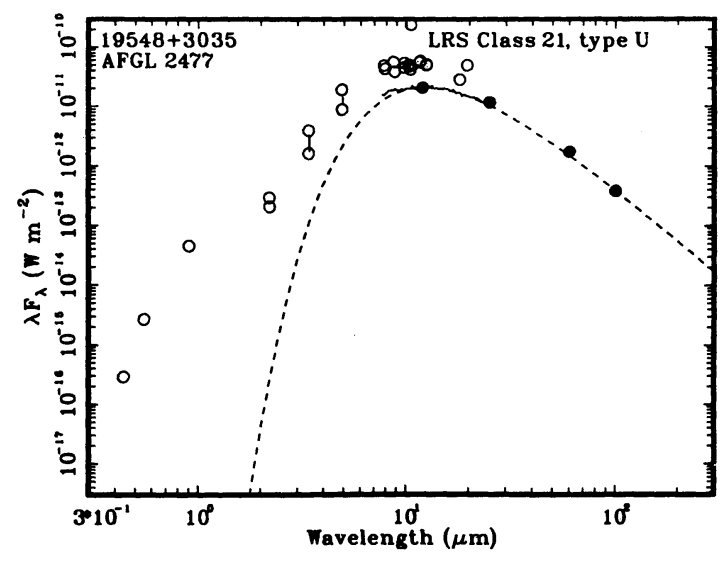

FIG. $2 b$

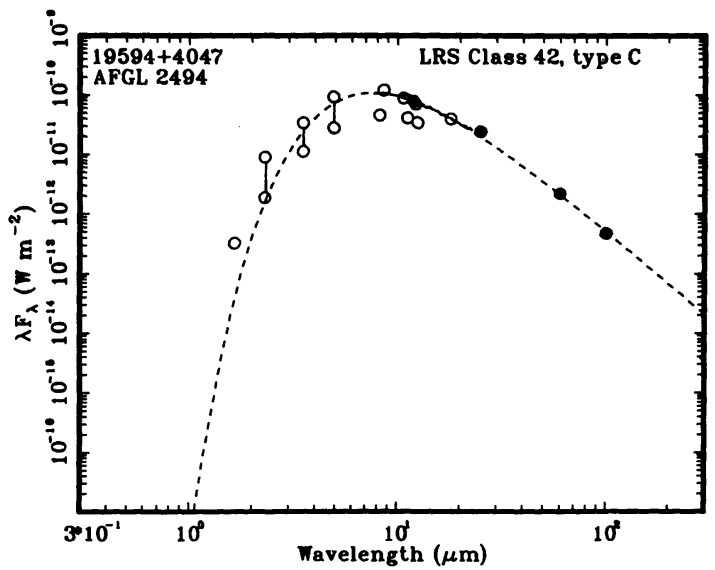

FIG. $2 d$

FIG. 2.-Energy distributions of four extreme carbon star candidates with existing ground-based data. The open circles are optical and infrared photometry in the literature. Measurements in the same band are joined by vertical lines to show the extent of variability. The filled circles are color-corrected $I R A S$ fluxes, and the IRAS LRS is shown as solid lines. Blackbody curves of $369,313,436$, and $296 \mathrm{~K}$ are plotted for $(a) 00210+6221,(b) 01144+6658,(c) 19548+3055$, and $(d)$ $19595+4047$, respectively.

\section{NEW INFRARED OBSERVATIONS}

Five of the sources in our extreme carbon star sample were observed at the United Kingdom Infrared Telescope (UKIRT) atop Mauna Kea, Hawaii, on 1990 August 21/22. The $3.8 \mathrm{~m}$ UKIRT was used in the $\mathrm{f} / 35.4$ configuration. The infrared detector employed was UKT8 (Ge:Ga bolometer) with the standard $L^{\prime}(3.4 \mu \mathrm{m}), M(4.7 \mu \mathrm{m}), N(10.5 \mu \mathrm{m}), Q(19.5 \mu \mathrm{m})$, and $Z(30 \mu \mathrm{m})$ filters. A 6".0 aperture was selected and an east-west throw of $20^{\prime \prime}$ at a frequency of $12.5 \mathrm{~Hz}$ was used. The beam was found to be circular with a FWHM of 5".5 by scanning the K2 star SAO 102476 at $10.5 \mu \mathrm{m}$.

Infrared standard stars were observed throughout each night to transform the carbon star observations to the standard system. The standards used were BS 337 ( $\beta$ And), BS 1457 $(\alpha$ Tau), and BS 7001 ( $\alpha$ Lyr). Extinction coefficients and zero points were calculated for each night. In each case the calculated extinction coefficients were within $1 \sigma$ of their median values (as determined by Krisciunus et al. 1987). Standard magnitudes were calculated using the nightly zero points and the median extinction coefficients. The results of the infrared photometry are given in Table 3 . The $Z$ filter measurements are more influenced by atmospheric conditions than the others because of the poor atmospheric window. Consequently the $Z$-magnitudes were not used for studying the energy distributions of the carbon stars, although the values are listed in Table 3.

In order to give the best chance for detection at mid-infrared wavelengths, we have selected a group of sources with $12 \mu \mathrm{m}$ flux densities greater than $100 \mathrm{Jy}$ and in the right ascension range $2^{\mathrm{h}}$ to $7^{\mathrm{h}}$. Subsequently $06582+1507$ was also observed because the IRAS data indicate it to be the most extreme object in the sample. The sources were located by searching around the IRAS positions in the $N$ band. The measured $N$ and $Q$ magnitudes were found to be consistent with the IRAS [12] and [25] magnitudes, confirming that the right objects had been found. No optical counterparts were found for $02293+5748,04530+4427$, and $06012+0726$ down to $V \sim 15$. $03448+4432$ has a faint optical counterpart. When $06582+1507$ was observed, dawn was rapidly approaching. The bright sky made it impossible to assess the existence of an optical counterpart and contributed to the large uncertainties in the observed magnitudes.

Since all these are very red objects with their flux distribution peaking around $10 \mu \mathrm{m}$, the UKIRT photometry was very 


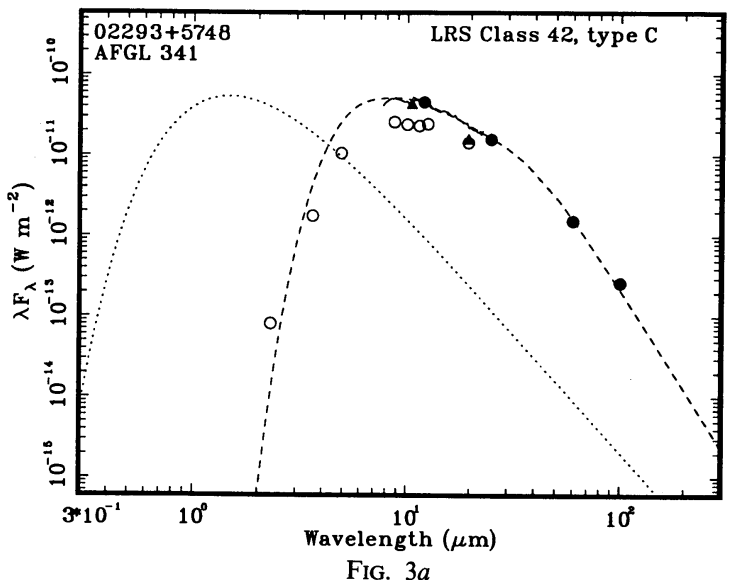

FIG. $3 a$

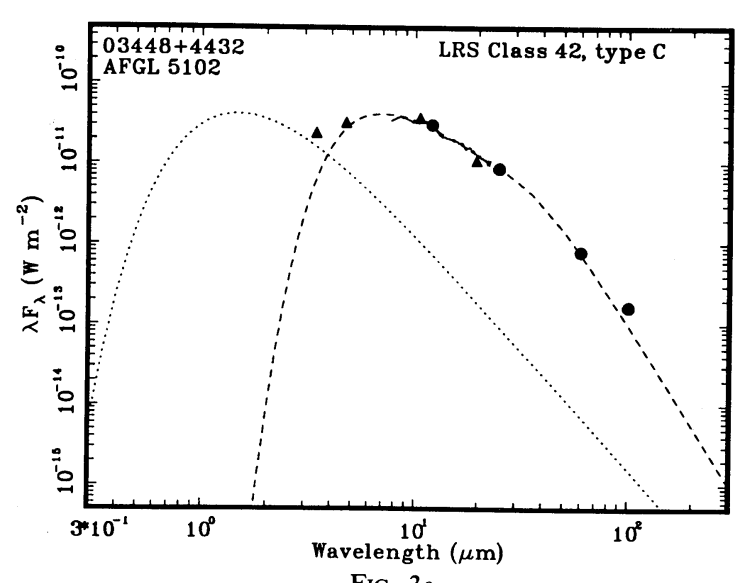

FIG. $3 c$

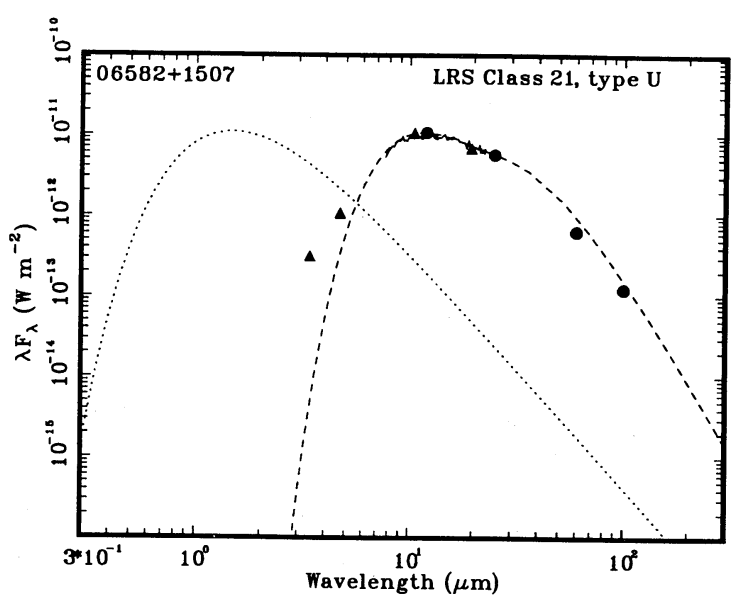

Fig. $3 e$

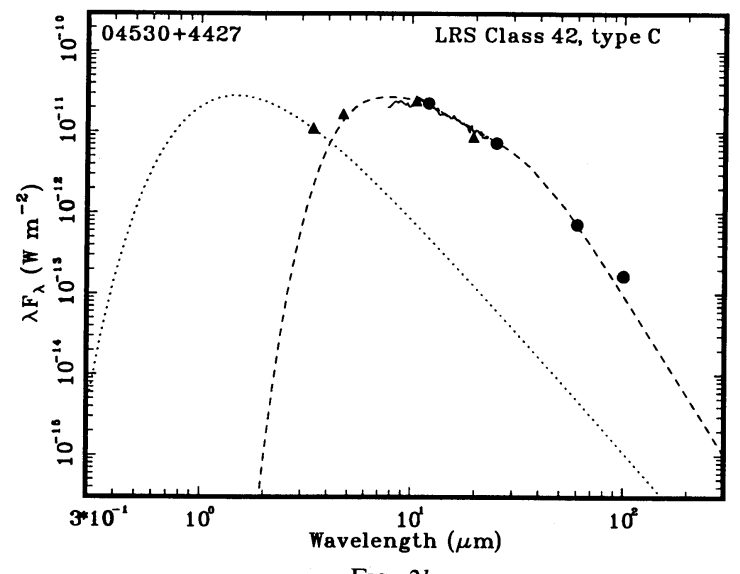

FIG. $3 b$

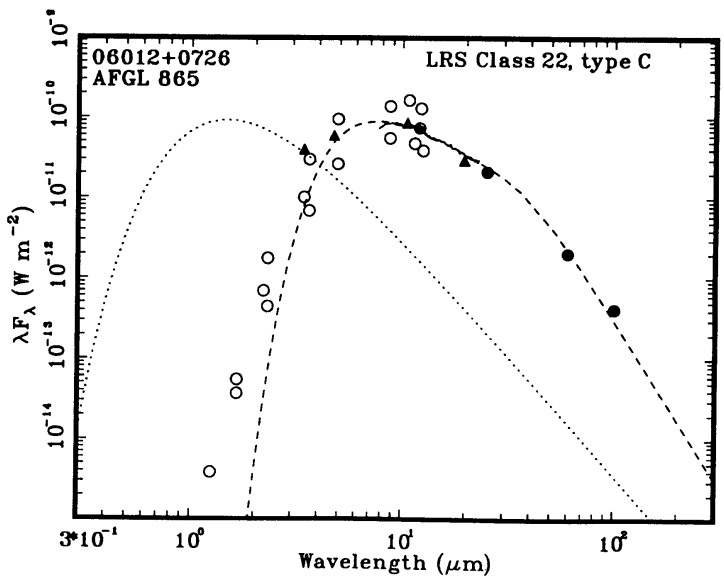

Fig. $3 d$

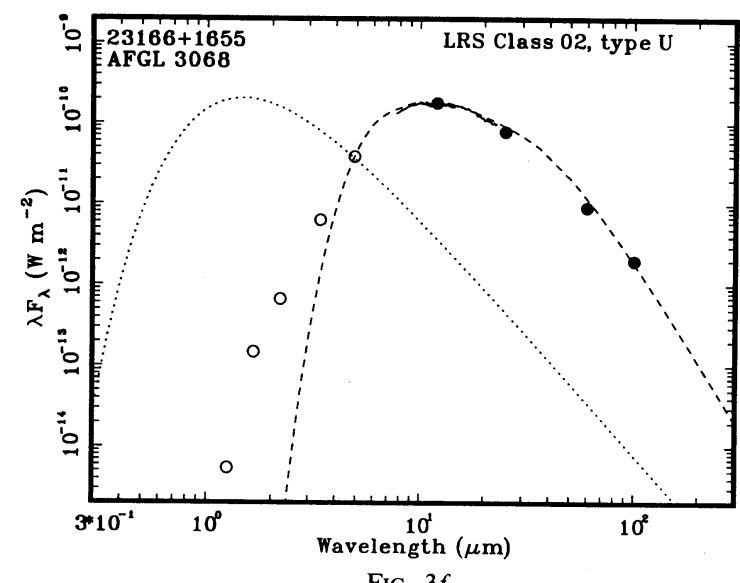

FIG. $3 f$

FIG. 3.-Energy distributions of five extreme carbon stars observed at UKIRT (plus AFGL 3068). The filled circles are color-corrected IRAS fluxes, the open

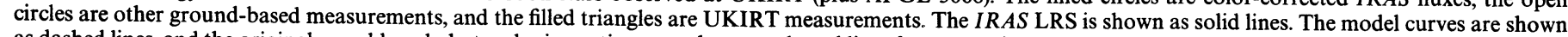
as dashed lines, and the original unreddened photospheric continua are shown as dotted lines for comparison.

useful in determining their overall energy distributions. The observed UKIRT data for the five sources are plotted in Figures $3 a-3 e$, together with their IRAS color-corrected fluxes. All five sources have color temperatures in the range of 300 to $500 \mathrm{~K}$. A similar plot for the AFGL 3068 is presented in Figure $3 f$ for comparison. Also plotted are the radiative transfer model fits (see $\S 5$ ) and the assumed photospheric continua of the central stars. We can see that all six stars are heavily reddened by circumstellar dust.

The LRS spectra of the five sources, plus AFGL 3068 as a prototype, are presented in Figure 4. IRAS $06582+1507$ has the lowest color temperature of the group, and has a completely featureless LRS spectrum. The other four candidates have some $11.3 \mu \mathrm{m}$ emission, strongest in $04530+4427$ and 
TABLE 2

CO OBSERVATIONS

\begin{tabular}{|c|c|c|c|}
\hline Name & $\begin{array}{l}\text { LRS } \\
\text { Class }\end{array}$ & $\begin{array}{c}\mathrm{CO} \\
\text { Transition }\end{array}$ & Reference \\
\hline $00210+6221$. & $\mathrm{U}$ & $1-0$ & Arquilla et al. 1986 \\
\hline $01144+6658 \ldots$ & $\mathrm{U}$ & $2-1$ & Zuckerman \& Dyck 1986a \\
\hline $02293+5748 \ldots \ldots \ldots \ldots$ & $\mathrm{C}$ & $2-1$ & Zuckerman et al. 1986 \\
\hline $03448+4432 \ldots \ldots \ldots \ldots$ & $\mathrm{C}$ & $2-1$ & Zuckerman \& Dyck 1986a \\
\hline $04530+4427 \ldots \ldots \ldots \ldots$ & $\mathrm{C}$ & $\begin{array}{l}1-0 \\
1-0\end{array}$ & $\begin{array}{l}\text { Zuckerman et al. } 1986 \\
\text { Nguyen et al. } 1987\end{array}$ \\
\hline $06012+0726 \ldots \ldots \ldots \ldots$ & $\mathrm{C}$ & $\begin{array}{l}1-0 \\
1-0 \\
2-1 \\
2-1\end{array}$ & $\begin{array}{l}\text { Knapp \& Morris } 1985 \\
\text { Zuckerman et al. } 1977 \\
\text { Knapp et al. } 1982 \\
\text { Wannier et al. } 1990\end{array}$ \\
\hline $08074-3615 \ldots \ldots \ldots \ldots$ & $\mathrm{U}$ & $1-0$ & Zuckerman \& Dyck 1986b \\
\hline $08171-2134 \ldots \ldots \ldots \ldots$ & $\mathrm{U}$ & $\begin{array}{l}1-0 \\
2-1\end{array}$ & $\begin{array}{l}\text { Zuckerman \& Dyck 1986b } \\
\text { Zuckerman \& Dyck } 1986 a\end{array}$ \\
\hline $17371-3021 \ldots \ldots \ldots \ldots$ & $\mathrm{C}$ & $1-0$ & Nguyen et al. 1987 \\
\hline $17534-3030 \ldots \ldots \ldots$ & $\mathrm{U}$ & $1-0$ & Zuckerman \& Dyck 1986b \\
\hline $19548+3035 \ldots \ldots \ldots \ldots$ & $\mathrm{U}$ & $2-1$ & Zuckerman \& Dyck 1989 \\
\hline $19594+4047 \ldots \ldots \ldots \ldots$ & $\mathrm{C}$ & $\begin{array}{l}1-0 \\
1-0\end{array}$ & $\begin{array}{l}\text { Zuckerman \& Dyck 1986a } \\
\text { Knapp (1986) }\end{array}$ \\
\hline $21318+5631 \ldots \ldots \ldots \ldots$ & $\mathrm{U}$ & $\begin{array}{l}1-0 \\
1-0\end{array}$ & $\begin{array}{l}\text { Zuckerman et al. } 1986 \\
\text { Lindqvist et al. } 1988\end{array}$ \\
\hline $21489+5301 \ldots \ldots \ldots \ldots$ & $\mathrm{C}$ & $2-1$ & Zuckerman \& Dyck 1986a \\
\hline AFGL $3068 \ldots \ldots \ldots \ldots$ & $\mathrm{U}$ & $\begin{array}{l}1-0 \\
1-0 \\
1-0 \\
2-1 \\
2-1\end{array}$ & $\begin{array}{l}\text { Zuckerman et al. } 1977 \\
\text { Zuckerman et al. } 1986 \\
\text { Knapp \& Morris } 1985 \\
\text { Knapp et al. } 1982 \\
\text { Olofsson et al. } 1987\end{array}$ \\
\hline
\end{tabular}

weakest in $06012+0726$. The feature strength does not appear to correlate with the slope of the continuum for these sources.

\section{EVOLUTIONARY STATUS OF EXTREME CARBON STARS}

Carbon-rich stars on the AGB can be divided into two groups based on their mid-infrared $(\sim 10 \mu \mathrm{m})$ properties: visual carbon stars whose infrared spectra are dominated by photospheric continuum, and infrared carbon stars whose infrared spectra are due to dust emission. The detection of $60 \mu \mathrm{m}$ excesses in visual carbon stars (Thronson et al. 1987) has led to the theory that visual carbon stars evolved from oxygenrich Mira variables, and are not undergoing any mass loss (Willems \& de Jong 1988; Chan \& Kwok 1988). Mass loss is assumed to initiate again after the formation of $\mathrm{SiC}$ grains, leading to infrared carbon stars showing the $11.3 \mu \mathrm{m} \mathrm{SiC}$ emission (Chan \& Kwok 1990). It is natural to assume that the extreme carbon stars under study here are just more evolved infrared carbon stars which have ascended further up the AGB and have higher luminosities and mass-loss rates. Figure 5 shows the distribution of 564, infrared carbon stars and the 31 extreme carbon star candidates in the IRAS color-color diagram. The extreme carbon star candidates seem to lie on the extension (toward lower temperatures) of the band occupied by infrared carbon stars.

While the colors of the stars in the present sample have a natural explanation, the question on the dust features is less clear. Those sources with weak SiC feature can be interpreted to be infrared carbon stars with such high optical depths in the circumstellar envelopes that the $11.3 \mu \mathrm{m}$ is partially in selfabsorption. For objects with no obvious features in the LRS, they could be stars with even higher mass-loss rates. The 11.3 $\mu \mathrm{m}$ feature may be just in between emission and absorption, leading to a featureless continuum. Infrared carbon stars that show the $11.3 \mu \mathrm{m} \mathrm{SiC}$ emission features can generally be very well fitted by standard radiative transfer models (Chan \& Kwok 1990). However, such models have difficulty in fitting the featureless objects here. In order to reproduce the red color of these objects (e.g., by having a very high mass-loss rate), the $\mathrm{SiC}$ feature would go into self-absorption. Indeed, there is no known case of clear, strong $11.3 \mu \mathrm{m}$ absorption in an LRS spectrum although there are a few possible candidates such as IRAS 16575 - 4023 (Volk et al. 1991).

An alternative explanation is that a dust grain other than $\mathrm{SiC}$ is responsible. For example, graphite grains are relatively featureless in the wavelength range of the LRS. Graphite, being a metal rather than a dielectric, would condense at lower temperatures than $\mathrm{SiC}$ (Gilman 1969). It is possible that when the star has evolved to lower temperatures near the tip of the AGB, graphite would condense in place of SiC.

We have carried out radiative transfer modeling to fit the available data for the five sources in Table 3 and for AFGL 3068. The modeling process involved constructing a series of trial models with different grain optical depths and density profiles proportional to $r^{-2}$ and $r^{-2.5}$ (corresponding to steady mass loss and mass loss increasing with time on the AGB; see Volk \& Kwok 1989) seeking a match to the IRAS colors. When a match was found, the LRS spectra for the source was compared to the model to see if the spectral shape and the strength of any features were correctly reproduced. In all of the models the central star was assumed to be a $2500 \mathrm{~K}$ blackbody. The exact temperature of the star is not important for the emergent spectrum for these sources because the dust shells are very optically thick. The modeling process is similar to that described in Volk \& Kwok (1988) except for the use of different grains in the models. Once the model parameters (dust optical

TABLE 3

INFRARED PHOTOMETRY

\begin{tabular}{|c|c|c|c|c|c|c|}
\hline \multirow[b]{2}{*}{ IRAS NAME } & \multicolumn{5}{|c|}{ UKIRT MAGNITUDES $^{\mathrm{a}}$} & \multirow{2}{*}{$\begin{array}{l}I R A S 12 \mu \mathrm{m} \\
\text { MAGNITUDES }\end{array}$} \\
\hline & $L^{\prime}$ & $M$ & $N$ & $Q$ & $Z$ & \\
\hline $02293+5748$ & & & -1.50 & -2.56 & & $-2.04 \pm 0.04$ \\
\hline $03448+4432$ & +2.51 & +1.22 & -1.33 & -2.15 & $-2.15 \pm 0.22$ & $-1.66 \pm 0.04$ \\
\hline $04530+4427$. & +3.33 & +1.93 & -0.88 & -1.91 & $-1.89 \pm 0.24$ & $-1.41 \pm 0.09$ \\
\hline $06012+0726 \ldots \ldots \ldots$ & +1.94 & +0.54 & -2.25 & -3.23 & $-3.26 \pm 0.17$ & $-2.62 \pm 0.05$ \\
\hline $06582+1507 \ldots \ldots \ldots$ & +7.23 & +4.94 & +0.04 & -1.64 & $-1.50 \pm 0.50$ & $-0.49 \pm 0.07$ \\
\hline
\end{tabular}

a Except for $06582+1507$, the uncertainties are $\pm 0.01 \mathrm{mag}$ in $L^{\prime}$ and $M, \pm 0.02 \mathrm{mag}$ in $N$ and \pm 0.03 mag in $Q$. For $06582+1507$, the uncertainties are \pm 0.1 in $L^{\prime} \pm 0.04$ in $M$, and \pm 0.03 for both $N$ and $Q$. The $Z$ filter magnitudes are much more uncertain due to poor atmospheric transmission at $30 \mu \mathrm{m}$. The IRAS color-corrected $12 \mu \mathrm{m}$ magnitudes are also listed for comparison.

${ }^{\mathrm{b}}$ IR AS magnitudes are as defined by Walker \& Cohen 1988. 

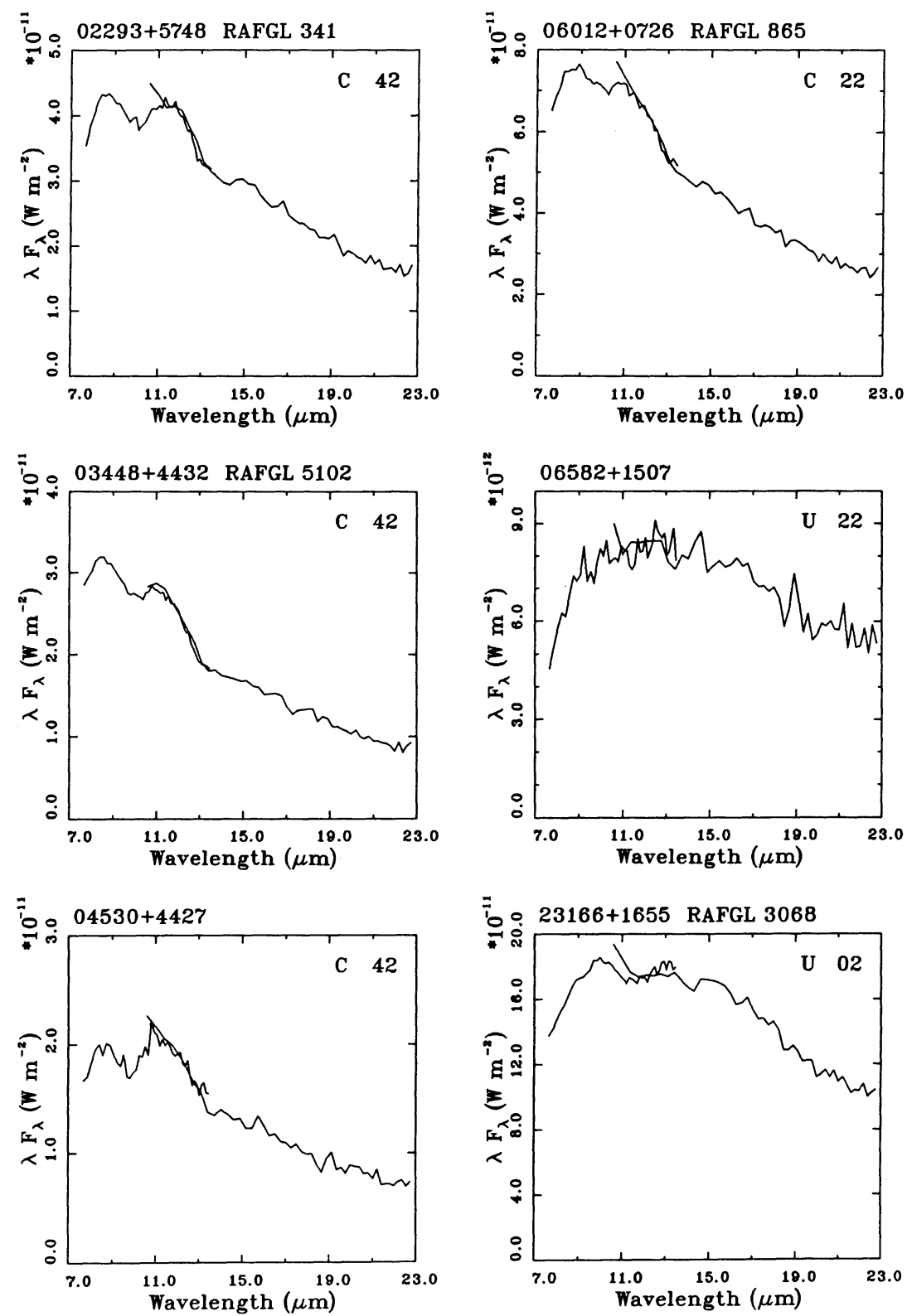

Fig. 4.-LRS spectra of five extreme carbon stars observed at UKIRT (plus AFGL 3068). The two-digit number on the top right-hand corner of each spectrum is the LRS Atlas classification. The letter codes (U or C) are spectral classification codes as defined by Volk \& Cohen (1989).

depth, dust condensation radius, and dust density profile) are determined, the gas mass-loss rate is derived using an assumed gas to dust ratio (Volk \& Kwok 1988).

Initial attempts to model the sources using the siliconcarbide opacity function of Chan \& Kwok (1990) were unsuccessful because the $11.3 \mu \mathrm{m}$ feature was too strong. Therefore a new set of models was carried out with a graphite-SiC mixture of $70 \%$ to $30 \%$ by mass with the graphite opacity function taken from Draine \& Lee (1984). This mixture proved able to reasonably account for five of the six sources. For $06582+1507$, which has extremely red IRAS colors and no hint of any features in the LRS spectrum it was necessary to use a pure graphite model of very high optical depth to match the LRS spectrum. In all of the graphite or mixture models the dust condensation temperature was assumed to be $1000 \mathrm{~K}$, and the dust and gas expansion velocities were assumed to be equal at $15 \mathrm{~km} \mathrm{~s}^{-1}$. In the models we assumed a gas to dust mass ratio of 260 for the graphite grains and 330 for the SiCgraphite grain mixture. The model parameters and the derived mass-loss rates are given in Table 4.

We also found that a steady mass-loss density profile predicts too much flux in the IRAS 60 and $100 \mu \mathrm{m}$ bands when the LRS spectrum was well fitted by the model. This suggests a steeper density gradient in the envelope and we found that a $r^{-2.5}$ density profile can fit the entire energy distribution. Such a density profile suggests that the mass-loss rate has been increasing on time scales of a few $10^{4} \mathrm{yr}$. The derived mass-loss rates given in Table 4 represent the mass-loss rate at present. The emergent spectra are then normalized to the observed data to yield the total observed flux (given in col. 6 of Table 4). The 
TABLE 4

Model AND Derived Parameters

\begin{tabular}{clcccc}
\hline \hline IR $A S$ Name & Dust & $(11.3 \mu \mathrm{m})$ & \multicolumn{1}{c}{$\begin{array}{c}\dot{M} / D \\
\left(M_{\odot} \mathrm{yr}^{-1} \mathrm{kpc}^{-1}\right)\end{array}$} & $\begin{array}{c}L_{*} / D^{2} \\
L_{\odot} \mathrm{kpc}^{-2}\end{array}$ \\
\hline $02293+5748 \ldots \ldots \ldots \ldots$ & mixture & 1.96 & 118 & $5.8(-6)$ & 2280 \\
$03448+4432 \ldots \ldots \ldots \ldots$ & mixture & 1.44 & 86 & $3.1(-6)$ & 1820 \\
$04530+4427 \ldots \ldots \ldots \ldots$ & mixture & 1.70 & 102 & $3.7(-6)$ & 1320 \\
$06012+0726 \ldots \ldots \ldots \ldots$ & mixture & 1.57 & 94 & $6.1(-6)$ & 4070 \\
$06582+1507 \ldots \ldots \ldots \ldots \ldots$ & graphite & 5.15 & 900 & $2.3(-5)$ & 472 \\
$23166+1655 \ldots \ldots \ldots \ldots$ & mixture & 7.85 & 471 & $3.3(-5)$ & 9420 \\
\hline
\end{tabular}

model fits are optimized to fit the peaks of the energy distributions (see Fig. 3), and especially the LRS spectra. All of the sources show a near-infrared excess over the models. However, such excesses represent only a small fraction of the total flux. We have been unable to account for the observed near-infrared excess and its origin remains a mystery.

The only other model fit for the infrared spectrum of AFGL 3068 is done by Rowan-Robinson \& Harris (1983). Their model derives a lower optical depth for AFGL 3068 because their model only passes through the $J$ band photometry but does not fit the longer wavelength data points. In our model, the emphasis is on fitting the LRS and the peak of the energy distribution. This results in a worse fit to the near-infrared photometry.

Table 5 presents the vertical distances of the sources from the Galactic plane $(z)$ in units of their distances. AFGL 3068 is at high Galactic latitude and is therefore unlikely to be more than $1 \mathrm{kpc}$ away. This puts an upper limit to its luminosity of $\sim 9500 L_{\odot}$. Of the other five sources in Table 5, 06582+1507 has a very low total flux, which implies a distance of several $\mathrm{kpc}$ if it were to have a luminosity typical of an evolved AGB star. This will put it at a large distance from the Galactic plane. Since a $z$ value of several hundred pc is very high for a star with a high main-sequence mass, it is more reasonable to assume that $06582+1507$ has a low main-sequence mass. Using the

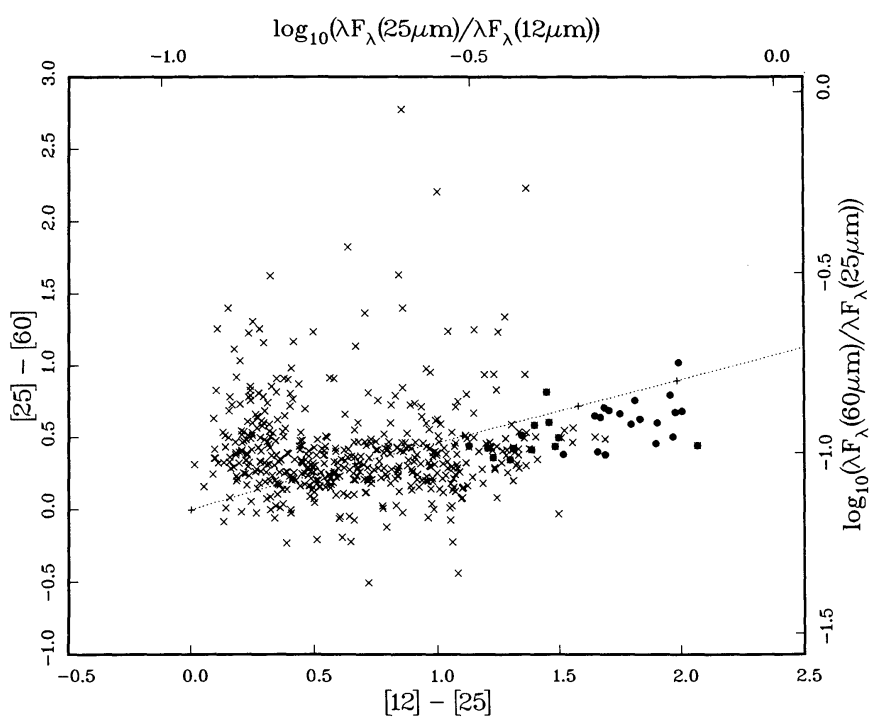

FIG. 5.-IRAS color-color diagram for the extreme carbon star sample. Sources in the extreme carbon star sample are shown as filled circles. Also plotted are the 564 stars $(x)$ in the LRS Atlas that are classified as Group C (11.3 $\mu \mathrm{m}$ emission). The blackbody curve is shown as a dotted line. The IRAS colors are as defined by Walker \& Cohen (1988). same reasoning, we can also argue for the low mass of $03448+4432$ and $06102+0725$. This is consistent with the suggestion of Bryan, Volk, \& Kwok (1990) that high-mass AGB stars stay oxygen-rich because the large envelope mass dilutes the amount of carbon dredged up from the core, and carbonstars mainly descend from a main-sequence population of lowmass stars. Further studies of these extreme carbon stars would be very valuable in determining the upper mass cutoff point for the formation of carbon stars.

\section{CONCLUSIONS}

From the IRAS LRS we have identified a group of extreme infrared carbon stars. The color temperatures of these candidates are lower than the most evolved infrared carbon stars known to date. Radiative transfer models constructed to fit the observed energy distributions suggest circumstellar extinction $\left(A_{v}\right)$ ranging as high as 900 for these stars. Such highly optically thick envelopes suggest that these candidates could be the most evolved carbon stars on the AGB.

While the red objects with weak $\mathrm{SiC}$ emission features can be interpreted as infrared carbon stars with the $\mathrm{SiC}$ partially in self-absorption, the featureless objects can only be fitted by introducing a featureless substance like graphite. This is consistent with the lower condensation temperature of graphite in comparison to $\mathrm{SiC}$, and graphite could be preferentially formed during the very last stage of the evolution of carbonrich stars on the AGB.

From the total observed fluxes and the galactic location of these extreme carbon star candidates, we estimate that their luminosities cannot exceed a few thousand solar luminosities otherwise they will be far off the plane. This suggests that they are less massive than the reddest $\mathrm{OH} / \mathrm{IR}$ stars $(\mathrm{OH} / \mathrm{IR}$ stars with silicate absorption features) which generally lie in the plane (Volk \& Kwok 1988; Likkel 1989). This supports the suggestion of Bryan et al. (1990) that there is an upper mass cutoff for the formation of carbon stars, above which the hydrogen envelope is too large for carbon dredge-up to overcome. It would be very interesting to further investigate the

TABLE 5

Galactic Distribution of THE Program Stars

\begin{tabular}{ccc}
\hline \hline Source Name & $b^{\mathrm{II}}$ & $z(\mathrm{pc}) / D(\mathrm{kpc})$ \\
\hline $02293+5748 \ldots \ldots \ldots$ & -2.22 & -39 \\
$03448+4432 \ldots \ldots \ldots$ & -7.62 & -133 \\
$04530+4427 \ldots \ldots \ldots$ & +0.88 & +15 \\
$06012+0726 \ldots \ldots \ldots$ & -7.03 & -123 \\
$06582+1507 \ldots \ldots \ldots$ & +8.91 & +155 \\
$23166+1655 \ldots \ldots \ldots$ & -40.35 & -648 \\
\hline
\end{tabular}


exact mass boundary for the branching between oxygen-rich and carbon-rich stars on the AGB.

We thank Bruce Hrivnak for assistance during the UKIRT observations. P. P. L. acknowledges the award of an NSERC postgraduate scholarship. This work is supported by the NASA Astrophysical Data Program grant 215-90. The University of Calgary IRAS Data Analysis Facility is supported by a grant from the Natural Sciences and Engineering Research Council of Canada.

\section{REFERENCES}

Arquilla, R., Leahy, D. A., \& Kwok, S. 1986, MNRAS, 220, 125

Atlas of Low Resolution IRAS Spectra 1986, IRAS Science Team, prepared by F. M. Olnon \& E. Raimond, A\&AS, 65, 607 (LRS Atlas)

Bryan, G. L., Volk, K., \& Kwok, S. 1990, ApJ, 365, 301

Chan, S. J., \& Kwok, S. 1988, ApJ, 334, 362 1990, A\&A, 237, 354

Draine, B. T., \& Lee, H. M. 1984, ApJ, 285, 89

Gaylard, M. J., West, M. E., Whitelock, P. A., \& Cohen, R. J. 1989, MNRAS, 236,247

Gehrz, R. D., \& Hackwell, J. A. 1976, ApJ, 206, L161

Gilman, R. C. 1969 , ApJ, 155, L185

Gosnell, T. R., Hudson, H., \& Puetter, R. C. 1979, AJ, 84,538

Hrivnak, B. J., Kwok, S., \& Boreiko, R. T. 1985, ApJ, 294, L113

IRAS Point Source Catalog, Version 2, 1988, Joint IRAS Science Working Group (Washington, DC: GPO) (PSC)

Jones, T. J., Bryja, C. O., Gehrz, R. D., Harrison, T. E., Johnson, J. J., Klebe, D. I., \& Lawrence, G. F. 1990, ApJS, 74, 785

Joyce, R. R., Capps, R. W., Gillett, F. C., Grasdalen, G. L., Kleinmann, S. G., \& Sargent, D. G. 1977, ApJ, 213, L125

Kleinmann, S. G., Gillett, F. C., \& Joyce, R. R. 1981, ARA\&A, 19, 411

Knapp, G. R. 1986, ApJ, 311, 731

Knapp, G. R., \& Morris, M. 1985, ApJ, 292, 640

Knapp, G. R., Phillips, T. G., Leighton, R. B., Lo, K. Y., Wannier, P. G., \& Wotten, H. A. 1982, ApJ, 252, 616

Krisciunus, K., et al. 1987, PASP, 99, 887

Kwok, S., Hrivnak, B. J., \& Boreika, R. T. 1987, ApJ, 312, 303

Leahy, D. A., Kwok, S., \& Arquilla, R. A. 1987, ApJ, 320, 825

Lebofsky, M. J., et al. 1978, ApJ, 219,487

Likkel, L. 1989, ApJ, 344, 350

Lindqvist, M., Nyman, L. A., Olofsson, H., \& Winnberg, A. 1988, A\&A, 205, L15

Little-Marenin, I. R., et al. 1987, AJ, 93, 663

Low, J. F., Kurtz, R. F., Vrba, F. J., \& Rieke, G. H. 1976, ApJ, 206, L153
Merrill, M., \& Stein, W. A. 1976, PASP, 88, 874

Neugebauer, G., \& Leighton, R. B. 1969, Two Micron Sky Survey, NASA SP-3047

Nguyen-Q-Rieu, Epchtein, N., Truong-Bach, \& Cohen, M. 1987, A\&A, 180, 117

Olofsson, H. 1987, in Late Stages of Stellar Evolution, eds. S. Kwok \& S. R. Pottasch (Dordrecht: Reidel), 149

Olofsson, H., Eriksson, K., \& Gustafsson, B. 1987, A\&A, 183, L13

Omont, A., Loup, C., Forveille, T., te Lintel Hekkert, P., \& Habing, H. 1990, in From Miras to Planetary Nebulae: Which Path of Evolution?, ed. M. O. Mennessier \& A. Omont (Gif-sur-Yvette: Editions Frontières), 301

Price, S. D., \& Walker, R. G. 1976, AFGL Rep. 76-0208 (Washington DC: GPO)

Rowan-Robinson, M., \& Harris, S. 1983, MNRAS, 202, 797

Thronson, H. A., Latter, W. B., Black, J. H., Bally, J., \& Hacking, P. 1987, ApJ, 322,770

Volk, K., \& Cohen, M. 1989, AJ, 98, 931

Volk, K., \& Kwok, S. 1988, ApJ, 331, 435

Volk. 1989, ApJ, 342, 345

Volk, K., Kwok, S., Stencel, R. E., \& Brugel, E. 1991, ApJS, 77, 607

Walker, H. J., \& Cohen, M. 1988, AJ, 95, 1801

Walker, H. J., Cohen, M., Volk, K., Wainscoat, R. J., \& Schwartz, D. E. 1989, AJ, 98,2163

Wannier, P. G., Sahai, R., Andersson, B.-G., \& Johnson, H. R. 1990, ApJ, 358, 251

Willems, F. J., \& de Jong, T. 1988, A\&A, 196, 173

Zuckerman, B., \& Dyck, H. M. 1986a, ApJ, 304, 394 . 1986b, ApJ, 311, 345

. 1989, A\&A,, 209, 119 Zuckerman, B., Dyck, H. M., \& Claussen, M. J. 1986, ApJ, 304, 401

Zuckerman, B., Palmer, P., Morris, M., Turner, B. E., Gilra, D. P., Bowers, P. F., \& Gilmore, W. 1977, ApJ, 211, L97 